\title{
Identifying and measuring stressors present in pre-hospital care
}

\author{
Peter Kindness, Prof. Chris Mellish \\ dot.rural, RCUK Digital Economy Research \\ University of Aberdeen \\ www.dotrural.ac.uk
}

\author{
Dr. Judith Masthoff \\ Computing Science Department \\ University of Aberdeen
}

\begin{abstract}
In this paper we introduce and outline the results of a survey designed to identify the demands and stressors experienced by pre-hospital care providers. We then present a controlled study that investigates how the stressors identified as being present in pre-hospital care can be measured using unobtrusive sensors measuring heart rate and skin conductance. We highlight how these findings can have important implications for future work regarding the development of systems for prehospital care providers.
\end{abstract}

Keywords- Stress, caregiver needs, unobtrusive sensors.

\section{INTRODUCTION}

The burgeoning research field of affective computing [12] combined with the development in sensor technologies presents the opportunity to investigate and react to the demands and stressors placed upon people entering 'critical situations'. In this paper we highlight the results of a survey exploring the concerns felt by carers in the pre-hospital domain (Section II) before detailing a study investigating how we can effectively measure these stressors using unobtrusive sensors (Section III). We conclude by outlining how these results provide a foundation upon which future work, investigating how stress reduction techniques can be applied to pre-hospital systems, can be based (Section IV).

\section{COMMUNITY FIRST RESPONDER SURVEY}

A Community First Responder (CFR) is a member of the public who volunteers to help their community by responding to medical emergencies while an ambulance is en route. CFRs receive basic emergency care training that enables them to provide limited treatment to the patient, before the arrival of the professional ambulance personnel. This service is particularly important in rural communities where the arrival of an ambulance may exceed the current target time in the United Kingdom of eight minutes [1]. The role of the CFR has been receiving considerable interest and support over recent years as ambulance services throughout the UK look to develop new strategies to help deliver and sustain emergency care, especially within rural communities [2], [3].

CFRs, like their professional counterparts, are entering situations where stressors are inherent, however as the notion of CFRs is relatively new there is a paucity of literature on which demands and stressors are experienced [4], [5]. We therefore developed and distributed, across Scotland, a survey aimed to measure the demands and stressors experienced by CFRs. A better understanding of which can aid the design of new systems being developed to assist CFRs.

\section{A. Design and distribution}

An established questionnaire designed by NASA for measuring workload, the NASA-TLX [6], formed the basis for our survey. Two key categories were identified for inclusion in our survey: 'demand' and 'emotion', each of which contained factors that could be measured. See Table I.

TABLE I. DEMANDS AND EMOTIONS

\begin{tabular}{|l|l|}
\hline \multicolumn{1}{|c|}{ Demands } & \multicolumn{1}{c|}{ Emotions of the Individual } \\
\hline Emotional (sympathy) & Frustration that you could not help more \\
\hline Mental (thinking) & $\begin{array}{l}\text { Worries about whether you are doing the } \\
\text { right thing }\end{array}$ \\
\hline Temporal (time pressure) & Irritation by external factors \\
\hline Physical & Being upset about (anticipated) outcome \\
\hline
\end{tabular}

CFRs rated to what extent these demands and emotions were present in both a typical callout and what they deemed to be the most stressful callout they had attended. Answers were recorded on a Likert scale of 1 to 10. Participants also ranked which of these demands and stressors they would consider to be the biggest cause of stress if present in a callout and which period of the callout they felt to be the most stressful.

The survey was distributed online by the Scottish Ambulance Service to CFR groups throughout Scotland to approximately 1000 CFRs at the time of participation.

\section{B. Survey results}

88 CFRs began the survey of which 40 continued through to completion. The results of the top demands and stressors experienced during a typical callout and what was deemed as being the most stressful callout is shown below in Table II.

Interestingly, when asked about what would be considered as the top demand or emotion (stressor) that attributes to stress if it were present, the emotion of feeling alone and isolated was regarded as the biggest (median: 8). This was true for prior to arrival, during the callout, and after handover to the paramedics. The time period that was deemed to be the most stressful was prior to arrival $(77.5 \%)$.

TABLE II. TOP DEMANDS AND STRESSORS - MEDIAN(IQR)

\begin{tabular}{|l|l|l|}
\cline { 2 - 3 } \multicolumn{1}{c|}{} & \multicolumn{1}{c|}{ Typical callout } & \multicolumn{1}{c|}{ Most stressful callout } \\
\hline Demand & Emotional $-7(3.5)$ & Mental $-7(3.5)$ \\
\hline Stressor & $\begin{array}{l}\text { Frustration that CFRs } \\
\text { could not help more }-6(6)\end{array}$ & $\begin{array}{l}\text { Frustration that CFRs } \\
\text { could not help more }-7(7)\end{array}$ \\
\hline
\end{tabular}




\section{Conclusions and findings}

The findings of the CFR survey [7] identified some of the emotional anxieties present amongst CFRs. These findings are a step towards understanding the needs of the pre-hospital care provider and should be taken into consideration when developing systems and interfaces designed to support CFRs. Due to the negative implications that stress could have for both care provider and receiver, it is important to consider the stress of the user in the design of such systems. However, before being able to effectively evaluate paradigms for managing stress, we first need to have an effective and feasible means of measuring the stressors identified. This motivates the following study for measuring stress using unobtrusive methods.

\section{MEASURING STRESS USING UNOBTRUSTIVE SENSORS}

\section{A. Study aims}

In this study we aim to investigate how stressors identified by CFRs can be represented using unobtrusive sensors. Selfreporting questionnaires have traditionally been used to measure stress, but these have several drawbacks. Some emotions can occur subconsciously [8] and questionnaires need to be completed post task. This, combined with the reluctance to disclose what some may deem as a weakness, cast doubt on the accuracy of self-reporting stress questionnaires. These existing questionnaires also tend to ask about events that occur over a long duration of time. This is not suitable for the measurement of acute stress, which occurs over a short duration of time. We utilise self-reported mood scales in this study, which can be applied over shorter time durations, to support data collected through more unobtrusive methods.

Physiological measurements can provide an insight into users' affective state without relying on cognitive judgments [9]. However, stress can have an impact on a person's physical, emotional, mental and behavioural states and can be easily misinterpreted and muddled with other phenomena, resulting in it being notoriously hard to measure. Until recently physiological measurements have relied on obtrusive and expensive apparatus. The development of increasingly unobtrusive sensors allows for the continuous measurement of users without interfering with the users primary task.

There are two major dimensions of emotion: arousal and valence [10]. Stress is categorised with both increased cardiovascular response (arousal) and increased negative emotion (valence) [8]. Stress can result in many physiological changes and as a result we will be measuring both heart rate and skin conductance to gauge user arousal [11], [12]. There are several methods for measuring valence such as a facial electromyograph [13] however these remain obtrusive. Because we are using a previously validated method of inducing stress (see Materials) we can be confident of negative valence. Despite this, we introduce the additional use of self-reporting mood questionnaires to ensure that participants are experiencing negative valence during the study which they consider to be stress and not for example anger. PANAS [14] is designed to assess participants' current feelings while the SACL [15] was developed to measure both the favourable or unfavourable appraisal of arousal, to gauge valence.

\section{B. Design}

A within subjects design was used, where participants performed tasks designed to induce moderate acute stress. Heart rate and skin conductance were recorded throughout.

\section{1) Participants}

13 participants were recruited from university students and staff (M:5, F:8, Mean Age(SD):27(7)). This number was based on a power calculation to avoid exposing too many people to unnecessary stress. Participants did not suffer from underlying health issues, and were 'vetted' for high neuroticism using a screening personality questionnaire [16]. None were excluded. We chose not to use CFRs in this early stage of research due the paucity and valuableness of this collective.

\section{2) Materials}

A slight adaptation of the Trier Social Stress Test (TSST) [17] was used. The TSST is a standardised protocol for the induction of moderate psychosocial stress in a laboratory setting. The TSST consists of several components which contribute to acute stress physiological responses including mental demand and anticipatory periods, both of which were identified as being the most stressful demands experienced by CFRs. The TSST satisfies the criteria of a motivated performance task that combines elements of uncontrollability and high levels of social-evaluative threat [18].

\section{a) Independent variable}

Time period: Briefing (TP1), Preparation time (TP2), Task (TP3) (which included an Interview (TP3a) and an Arithmetic Task (TP3b)) and Debriefing (TP4). See Figure 1.

\section{b) Dependent variables}

Mean heart rate: Heart rate was measured with the Garmin Forerunner 305 [19], using a sensor attached around the participant's chest. We measured the mean heart rate (in bpm).

Mean skin conductivity: Skin conductance was measured using the Q sensor [20]. The sensor attaches to the wrist and has been shown to record skin conductivity as effectively as traditional obtrusive apparatus [21]. For the Q sensor, skin conductance level was reported to rise in response to stressors such as cognitive load and physical exertion. We look to verify this with the validated TSST and investigate its relationship with heart rate. We measure mean skin conductance (in $\mu \mathrm{s}$ ).

Questionnaires: We used the PANAS to measure mood and the SACL to measure the favourable or unfavourable appraisal of arousal.

TABLE III. MEAN (SD) OF VALUES FOR HEART RATE AND SKIN CONDUCTIVITY

\begin{tabular}{|l|c|c|c|c|c|c|}
\cline { 4 - 6 } \multicolumn{1}{c|}{} & \multirow{2}{*}{ TP1 } & \multirow{2}{*}{ TP2 } & \multicolumn{3}{c|}{ TP3 } & \multirow{2}{*}{ TP4 } \\
\cline { 4 - 6 } \multicolumn{1}{c|}{} & & & TP3a & TP3b & Overall & \\
\hline Heart Rate $(\mathbf{b p m})$ & $85.1(12.5)$ & $95.7(16.2)$ & $105.4(21.1)$ & $94.8(14.7)$ & $100.1(17.4)$ & $80.8(12.5)$ \\
\hline Skin Conductance $(\boldsymbol{\mu s})$ & $0.77(0.86)$ & $1.10(1.22)$ & $2.09(1.98)$ & $2.45(2.19)$ & $2.26(2.06)$ & $2.02(1.81)$ \\
\hline
\end{tabular}




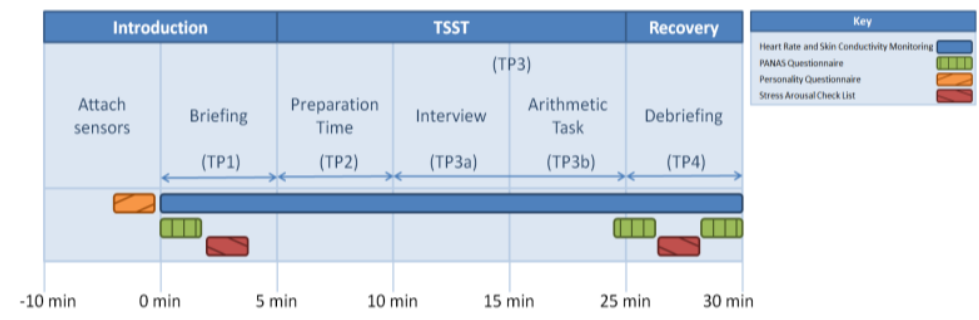

Figure 1. Study overview

\section{3) Hypotheses}

H1: There will be a significant effect of time period on the mean heart rate.

H1.1: Mean heart rate in time period TP1 will be significantly lower than in time period TP2.

H1.2: Mean heart rate in TP3a and TP3b will be significantly higher than in TP2.

H2. There will be a significant effect of time period on the mean skin conductance level.

H2.1: Mean skin conductance in TP1 will be significantly lower than in TP2.

H2.2: Mean skin conductance in TP3a and TP3b will be significantly higher than in TP2.

H3. Negative mood will be higher at the end of TP3 than that measured at TP1.

H4. SACL scores for stress at the end of TP3 will be higher than during TP1.

H5. Means of heart rate and skin conductivity will be a highly correlated with negative mood and stress scores.

\section{Results}

\section{1) Heart Rate}

One participant was excluded due incomplete sensor readings. There was a significant effect of time period on heart rate (GLM Repeated measures, Greenhouse-Geisser, F(2.37)= 14.23, $\mathrm{p}<.001)$, see Table III. This confirms H1. Using pairwise comparisons, the mean heart rate in TP1 was significantly lower than in TP2 (Bonferroni corrected, $\mathrm{p}<.01$ ). This also confirms H1.1. However, mean heart rate was not significantly higher in TP3a and $3 b$ than in TP2. So, there is no support for H1.2. We had assumed that time period TP3 (where participants performed the interview and arithmetic tasks) would be the most stressful and thus produce the highest heart rate. However preparation seems to have been equally stressful. In hindsight, the time pressure of having to prepare a presentation within three minutes is also a stressor [22].

\section{2) Skin conductance}

There was a significant effect of time period on the mean skin conductivity (GLM Repeated measures, GreenhouseGeisser, $\mathrm{F}(1.64)=10.2$, $\mathrm{p}<.005)$, see Table III. This confirms $\mathrm{H} 2$. Although skin conductivity was higher in TP2 than TP1, this was not significant. So, there is no support for H2.1. Unlike the conclusions drawn for $\mathrm{H} 1.2$ regarding heart rate, skin conductivity was shown to be significantly higher in TP3a and $3 \mathrm{~b}$ than in TP2 (Bonferroni corrected, $\mathrm{p}<.05$ ), confirming $\mathrm{H} 2.2$.

Figure 2 displays data gathered from the same person. The majority of graphs were similar to the one shown here. It is clear that heart rate reacts to stressful situations much more quickly than skin conductivity. When heart rate is at its highest at 6 minutes in, skin conductivity is still on the rise and does not peak until the debriefing period (during TP4) 20 minutes into the study. This is despite this period recording the lowest heart rate and the lowest scores in the self-reporting (see below). This explains the differences we found in the statistical analysis for heart rate and skin conductivity. The lack of support for $\mathrm{H} 2.1$ compared to the support for $\mathrm{H} 1.1$ seems to be due to the skin conductivity lagging behind. Determining the exact duration of emotions is a notoriously hard problem [23]. We tried to combat this with the time-stamping of the data to distinguish between time periods. This is corroborated by the support for H2.2 while there was no support for H1.2.

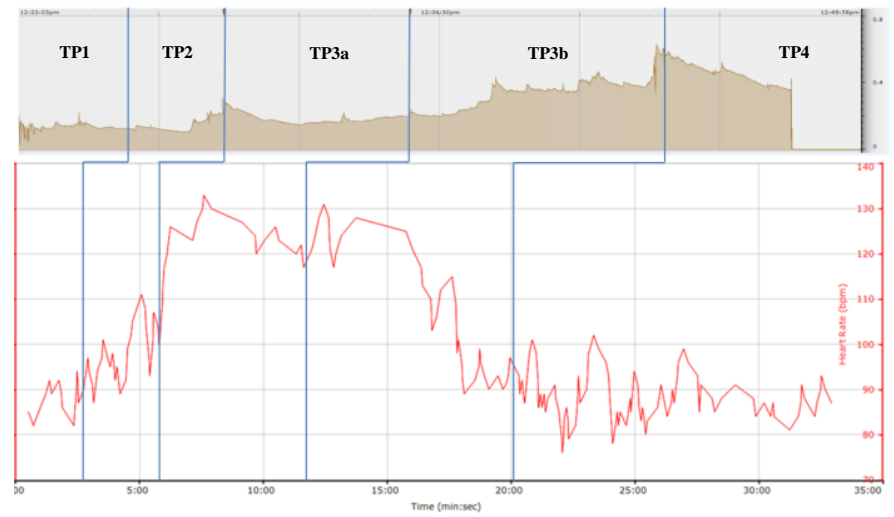

Figure 2. Output of skin conductivity (top graph) and heart rate (bottom graph) of a typical participant

\section{3) Mood}

The self-reported negative mood was significantly lower during the briefing (TP1) than directly after the task (end of TP3) ( $\mathrm{p}<.01)$, see Table IV below. This supports H3.

TABLE IV. MEAN (SD) OF SELF REPORTING QUESTIONNAIRES

\begin{tabular}{|l|l|l|l|}
\cline { 2 - 4 } \multicolumn{1}{c|}{} & \multicolumn{1}{c|}{ TP1 } & \multicolumn{1}{c|}{ TP3 } & \multicolumn{1}{c|}{ TP4 } \\
\hline Positive Mood & $30.69(8.23)$ & $28.62(8.92)$ & $29.85(8.27)$ \\
\hline Negative Mood & $14.62(2.93)$ & $21.31(6.90)$ & $13.85(4.08)$ \\
\hline Stress & $2.54(2.73)$ & $6.23(3.54)$ & $1.46(2.31)$ \\
\hline Arousal & $6.692(2.10)$ & $5.46(2.30)$ & $6.69(1.75)$ \\
\hline
\end{tabular}




\section{4) Stress and arousal}

The self-reported stress was significantly lower in the briefing (TP1) than directly after the task (end of TP3) and after debriefing (TP4) (Bonferroni corrected, $\mathrm{p}<.05$ ). This supports H4. However no significant result was found for arousal between the time periods as shown in Table IV. We did not have a hypothesis related to arousal as we focused on the stress scores from the SACL, but note that arousal did not increase, which supports that we are indeed measuring stress.

\section{5) Comparisons between measurements}

The two self-reporting measures were strongly, significantly and positively correlated for task and debriefing (TP3: Pearson correlation $=.7, \quad \mathrm{p}<.01$ and TP4: Pearson correlation=.73, $\mathrm{p}<.01$ ). However there was no correlation between mean heart rate, skin conductivity and self-reporting for any time period. Therefore H5 does not hold. This seems to indicate that you can measure stress but not the intensity of stress using this approach. This is likely due to the problem of accurately matching sensor data to the occurrence of specific events (sensor lag).

\section{6) Discussion}

There are several conclusions that can be drawn from this study. Most interesting for future work is the effect acute stress has on skin conductivity compared to heart rate. The results gathered in this study show that the reaction to stress shown by skin conductivity lags considerably behind the reaction shown by heart rate. This means that although skin conductance has been shown to measure stress, it needs to have a substantial period of time before and after the stressor for results to be clearly shown. It appears that skin conductance is more suited to long term monitoring of stress and that to get a fast response, to acute stress using unobtrusive sensors, heart rate is a better option. The results gathered through traditional self-reporting support that the response shown by the sensors was indicating stress rather than another phenomenon. However, it may still be important to measure skin conductance in association with heart rate to gauge a more accurate measurement of arousal, as long as sufficient intervals between stressors are introduced.

Various unforeseen problems arose whilst carrying out this study. The reliability of establishing a secure sensor fit in order to obtain accurate measurements on a variety of participants builds was one. Results also showed that skin conductivity between participants varied greatly although the overall trends were similar. This could be due to sensor tightness, positioning of the sensor or just different temperament between participants. These issues were experienced within a controlled environment and emphasize the challenges of using sensors in the 'real world'. These variables all add to the difficulties of defining a common stress model across all participants and interpreting physiological data remains a challenging problem.

\section{CONCLUSIONS AND IMPLICATIONS}

In this paper, we have highlighted a survey that explored the demands and stressors experienced by CFRs whilst they attended to callouts. We then detailed a study investigating how stress can be represented using unobtrusive health sensors. The ability to induce and measure the stressors which CFRs identified, allows for the evaluation of different stress reducing techniques and paradigms. The ability to pervasively monitor the emotional reactions to such methods is a significant step towards prompting the welfare of users entering critical, and inherently stressful situations. In future studies we investigate the effects that a virtual teammate might have on the stressor of loneliness.

\section{ACKNOWLEDGMENT}

We thank the Scottish Ambulance Service for their cooperation. This research is supported by the RCUK dot.rural Digital Economy Research Hub, University of Aberdeen.

\section{REFERENCES}

[1] Scottish Ambulance Service 2012-13 HEAT delivery plan, (http://goo.gl/BHIuy).

[2] Healthcare Commission. "The Role and Management of Community First Responders". London: Commission for Healthcare Audit and Inspection, 2007.

[3] SAS Community Resilience Strategy 2011-2015, (http://goo.gl/kzNpJ).

[4] S. Timmons, \& A. Vernon-Evans, "Why do people volunteer for community first responder groups?” Emergency Medicine Journal, 2012.

[5] E. Davies, et al. "Public access defibrillation: psychological consequences in responders." Resuscitation 77.2, pp. 201-206, 2008.

[6] S. Hart, \& L. Staveland, "Development of NASA-TLX (Task Load Index): Results of empirical and theoretical research". In P. A. Hancock and N. Meshkati (Eds.) Human Mental Workload, 1988.

[7] P. Kindness, et al. "The need to address stress: an insight into the demands and stressors experienced by first responders", unpublished.

[8] P. Feldman, et al. "Negative emotions and acute physiological responses to stress", Annals of Behavioral Medicine, 21, pp. 216-222, 1999.

[9] R. Picard, \& S. Daily, "Evaluating affective interactions: alternatives to asking what users feel", CHI-05 Wksp on Evaluating Affective Interfaces: Innovative Approaches, 2005.

[10] P. Lang, "The emotion probe: Studies of motivation and attention", American Psychologist, 50(5), pp. 372-385, 1995.

[11] H. Prendinger, "A study in users' physiological response to an empathic interface agent", Int. J. of Humanoid robotics. 3, pp. 371-391, 2006.

[12] R. Picard, "Affective Computing", MIT, 1997.

[13] R. Hazlett, "Measuring emotional valence during interactive experiences: boys at video game play", In Proceedings of the SIGCHI conference on Human Factors in computing systems, pp. 1023-1026, 2006.

[14] D. Watson, et al. "Development and validation of brief measures of positive and negative affect: the PANAS scales", J. of personality and social psych., 54, pp. 1063-1070, 1988.

[15] M. King, et al., "Measurement of stress and arousal", British Journal of Psychology, 74, pp. 473-479, 1983.

[16] S. Gosling, P. Rentfrow, \& W. Swann, "A very brief measure of the BigFive personality domains", Journal of Research in personality, 37, pp. 504-528, 2003.

[17] C. Kirschbaum, K. Pirke, \& D. Hellhammer, "The 'Trier Social Stress Test'”, Neuropsychobiology, 28, pp. 76-81, 1993.

[18] S. Dickerson, \& M. Kemeny, "Acute stressors and cortisol responses: a theoretical integration and synthesis of laboratory research", 130, pp. 355-391, 2004

[19] Garmin Forerunner 305 (http://goo.gl/d9U21).

[20] Affectiva Q sensor (www.affectiva.com/q-sensor).

[21] M. Poh, N. Swenson, \& R. Picard, "A wearable sensor for unobtrusive, long-term assessment of electrodermal activity" IEEE Transactions on Biomedical Engineering, 57, pp. 1243-1252, 2010.

[22] O. Svenson, "Time pressure and stress in human judgment and decision making", Springer, 1993.

[23] R. Levenson, "Emotion and the autonomic nervous system." Social Psychophysiology and Emotion: Theory and Clinical Appl., pp. 12-72, 1988. 